\title{
Kompetenzorientierte Gestaltung mobiler Lernfabrikmodule bei der DB Netz AG
}

\author{
Antonio Kreß, \\ Nicole Lieb, \\ Verena Lorenz und \\ Joachim Metternich, Darmstadt
}

Die Idee zur Konzeption einer mobilen Lernfabrik ergibt sich aus der Motivation, eine praxisnahe Lernumgebung für das operative Instandhaltungspersonal zu schaffen. Der vorliegende Beitrag beschreibt die kompetenzorientierte Gestaltung mobiler Lernfabrikmodule für die DB Netz AG. Dazu wurden drei mobile Lernfabrikmodule entwickelt, die die Themen Arbeitsorganisation, gewerkeübergreifende Systembetrachtung und Arbeitssicherheit behandeln. Das zugrundeliegende dreijährige Forschungsprojekt wird durch eine Kooperation zwischen der DB Netz AG und der TU Darmstadt ermöglicht.

gen durchzuführen. Im Rahmen eines dreijährigen Forschungsprojekts mit dem PTW der Technischen Universität Darmstadt soll in zwei Ausbaustufen eine Lernfabrik für die DB Netz AG entwickelt und konfiguriert werden. In der ersten Stufe werden dafür mobile Lernfabrik-Module entwickelt, die in diesem Beitrag beschrieben werden. Die zweite Stufe fokussiert die Konfiguration einer standortgebundenen Lernfabrik.

\section{| Konzept Lernfabrik}

ent, Industrie 4.0 und Energiee zienz [1]. Bereits seit 2007 wird die Prozesslernfabrik der Technischen Universität Darmstadt „Center für industrielle Produktivität“ durch das Institut für Produktionsmanagement, Technologie und Werkzeugmaschinen (PTW) betrieben. Seitdem wurden durch das PTW weltweit Lernfabriken aufgebaut, sodass entsprechende Erfolge vorhanden sind [2].

Die DB Netz AG ist für die Instandhaltung des größten Schienennetzes in Europa verantwortlich. Täglich fahren 40.000 Züge auf dem Schienensystem der DB Netz AG. Der Umsatz im Jahr 2017 betrug 5,6 Milliarden Euro. Die Entwicklung von Kompetenzen spielt auch für die DB Netz AG, die europaweit über 41.000 Mitarbeitende beschäftigt, eine wesentliche Rolle. Die Idee einer Lernfabrik entstand aus der Motivation, für das operative Instandhaltungspersonal eine erlebbare und praxisnahe Lernumgebung zu gestalten, um kompetenzorientierte Schulun-
Gegenüber klassischen Lehr-Lern-Methoden bieten Lernfabriken vielfältige Vorteile, indem sie Lernende durch einen höheren Bezug zum beruflichen Alltag motivieren. Dies erleichtert den Transfer in den Alltag. Durch die Anwendung von gelerntem Wissen in einem sicheren Umfeld übernimmt der Lernende eine aktive Rolle im Lernprozess. Das selbstständige Lösen von realen Problemen wird ermöglicht und damit die Entwicklung von Kompetenzen gefördert. Außerdem bieten Lernfabriken die Möglichkeit, neue Prozesse, Technologien und Methoden in einem sicheren Umfeld zu testen [3].

Aus diesen Gründen verbreiten sich Lernfabriken derzeit weltweit. Dabei ist zu erkennen, dass sich ein Großteil der betriebenen Lernfabriken in Europa befindet. Schwerpunkte bilden u.a. die Themen Lean Management, Industrie 4.0 und Energieeffizienz. Lernfabrik-Betrei- ber sind vor allem Universitäten. Jedoch bauen Unternehmen in den letzten Jahren vermehrt Lernfabriken auf, um die Kompetenzen eigener Mitarbeitende zu schulen und die oben beschriebenen Vorteile zu nutzen [1].

Bei Lernfabriken handelt es sich um Fabriken, in denen Forschung, Lehre und Weiterbildung stattfinden [4]. Lernfabriken sind gekennzeichnet durch folgende Merkmale [4]:

authentische Prozesse mit mehreren Stationen,

- eine Umgebung, die an einem realen Wertstrom angelehnt ist,

- ein physisches Produkt,

- ein didaktisches Konzept, das eigenständige Handlungen ermöglicht,

- eine klare Zielsetzung hinsichtlich ihres Schwerpunkts sowie

- ein nachhaltiges Betreibermodell.

Lernfabriken im weiteren Sinne beinhalten außerdem Konzepte des virtuellen Lernens, Fernunterricht und haben das Erzeugen einer Dienstleistung statt eines physischen Produkts zum Gegenstand. Bei der für die DB Netz AG zu entwickelnde Lernfabrik wird die Organisation des Instandhaltungsprozesses als Dienstleistung im Mittelpunkt stehen. Gleichzeitig soll die hier vorgestellte Lernfabrik mobil sein. Die einzelnen Punkte zur Definition von Lernfabriken werden auch für ein mobiles Konzept erfüllt. Deshalb wird im Folgenden von einer mobilen Lernfabrik gesprochen. 


\section{Methodik zur Lernfabrik-Gestaltung}

Um Lernfabriken zu gestalten, werden drei Ebenen betrachtet: die Makro-, Meso- und Mikro-Ebene. In der MakroEbene stehen die Lernfabrik-Infrastruktur und das Curriculum mit den Lernzielen im Fokus. Die Meso-Ebene beschäftigt sich mit der Gestaltung von Lernmodulen, die in der Mikro-Ebene auf einzelne Lehr-Lern-Situationen detailliert werden. Die Gestaltung von Lernfabriken teilt sich außerdem in zwei didaktische Transformationen auf (Bild 1). In der ersten didaktischen Transformation wird der Kompetenzbedarf anhand der Zielgruppe, den Unternehmenszielen und den organisatorischen Rahmenbedingungen analysiert. Die zweite didaktische Transformation behandelt die Gestaltung der technischen Infrastruktur und des Lernprozesses [5].

Zunächst wurden die organisationalen Anforderungen und der Kompetenzbedarf für die DB Netz AG ermittelt. Unternehmensziele leiten sich aus den Bereichen Qualität, Kosten, Zeit, Flexibilität, Humanität sowie unternehmensindividuellen Ziele ab. Zur Ableitung der intendierten Kompetenzen wurden umfangreiche Experteninterviews mit internen Trainern, Fachexperten und Weiterbildungspersonalen geführt. Die Zielgruppe besteht aus operativen Mitarbeitenden bis zur Teamleiterebene. Inhaltlich stammen die Kompetenzen und Lernziele aus den Gestaltungsprinzipien und Methoden der schlanken Produktion [6] und aus Trainerleitfäden bestehender Lernmodule. Zusätzlich wurden Experteninterviews geführt. Um die Wichtigkeit der intendierten Kompetenzen zu bestimmen, wurden diese mit den Unternehmenszielen der DB Netz AG in einer Matrix verknüpft und bewertet (Bild 2). Die Gewichtung der Unternehmensziele ist dabei durch die DB Netz AG vorgenommen worden. Die Wichtigkeit der Kompetenzen bestimmt sich aus der Summe der Gewichtungen der Unternehmensziele, die durch die Kompetenz beeinflusst werden. Außerdem wurde analysiert, welche Kompetenzen über ein mobiles Konzept geeignet sind. Auf diese Weise konnten die Kompetenzen identifiziert werden, die durch die mobile Lernfabrik gefördert werden können.

Nach der Formulierung der einzelnen Kompetenzen über Taxonomiestufen [7] wurden konkrete Trainingsszenarien mit-
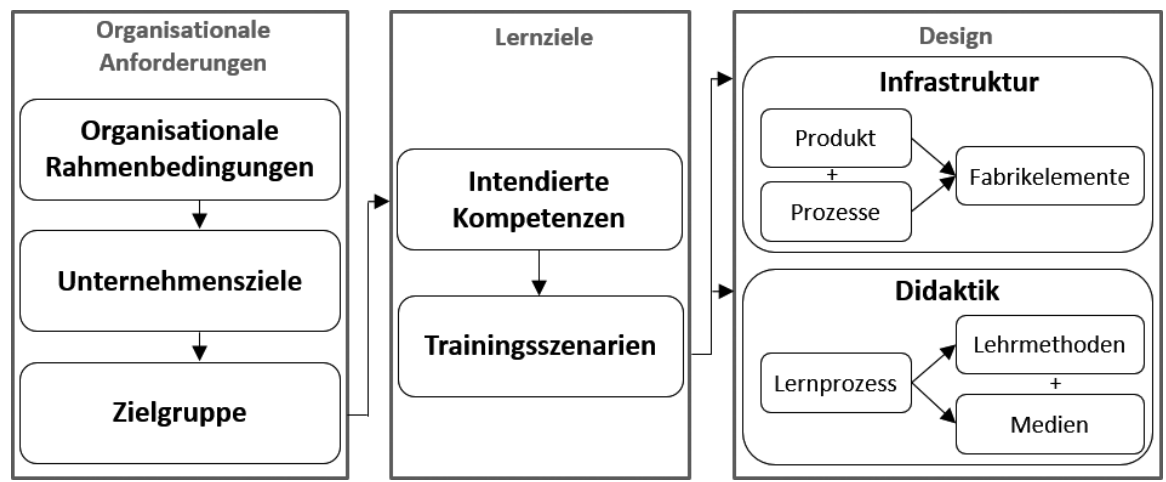

Bild 1. Modell zur kompetenzbasierten Gestaltung von Lernfabriken (i. A. an [6]) hilfe von Wissens- und Handlungselementen erstellt [8]. Mit den Übungsszenarien wurden die physische Infrastruktur sowie der Lernprozess gestaltet. Im Anschluss evaluierten Fachexperten und Trainer die entwickelten Lernmodule mehrfach. Dazu wurden die Lernmodule jeweils praktisch umgesetzt. Das durch Fragebögen und persönliche Gespräche aufgenommene Feedback wurde iterativ implementiert, um die Lernmodule zu verbessern.

\section{| Ergebnis}

Aus der Analyse des Kompetenzbedarfs ergeben sich 55 Kompetenzen. Nach der Filterung für ein mobiles Konzept konnten die wichtigsten Kompetenzen zu den Themen Arbeitsorganisation, Systembild Instandhaltung und Arbeitssicherheit geclustert werden. Das erstellte Ziele-Inhalt-Methoden (ZIM)-Papier bietet umfassende Hilfestellungen für die Unterrichtenden. Ein ZIM-Papier zeigt zu jeder Teilkompetenz (Ziel) die inhaltliche Gestaltung der Lehr-Lern-Situation (Inhalt) und die methodischen Umsetzung (Methode/Material). Die Teilkompetenzen sind dabei nach dem zeitlichen Lernmodul-Ablauf geordnet. Die Unterlagen in- klusive verschiedener Checklisten sollten so gestaltet sein, dass eine standardisierte Durchführung so einfach wie möglich für den Unterrichtenden ist. Die Lernmodule werden im Folgenden beschrieben:

Im ersten Modul lernen die Teilnehmenden Methoden aus dem Bereich Lean Management kennen und wenden diese an einer Modelleisenbahn an. Die Modelleisenbahn wird zu Beginn anhand eines Gleisplans (Bild 3) in Gruppenarbeit aufgebaut. Im Anschluss diskutieren die Teilnehmenden mögliche Verbesserungsmaßnahmen zum Aufbau. Diese werden durch praxisnahe Einblicke in die Verschwendungsarten sowie der Methode 5S ergänzt. Nach der Implementierung der Verbesserungsmaßnahmen bauen die Teilnehmenden den Gleisplan erneut auf und erkennen den Erfolg ihrer Maßnahmen anhand der kürzeren Aufbauzeit, besseren Absprachen und einem strukturierteren Vorgehen. Außerdem verstehen die Teilnehmenden die Bedeutung von Kennzahlen, wie z.B. der Meldequalität, anhand ausgewählter Beispiele.

Das zweite Lernmodul vertieft das Verständnis für das Gesamtsystem Instandhaltung. Die Teilnehmenden erkennen

\begin{tabular}{|c|c|c|c|c|c|c|}
\hline \multirow[t]{2}{*}{ Unternehmensziele } & \multirow{2}{*}{$\begin{array}{l}\text { Gewichtung } \\
\text { [1-5] }\end{array}$} & \multicolumn{5}{|c|}{ Intendierte Kompetenzen } \\
\hline & & Methode 5S & $\begin{array}{c}\text { Systemwissen } \\
\text { IH }\end{array}$ & $\begin{array}{l}\text { Arbeits- } \\
\text { sicherheit }\end{array}$ & $\ldots$ & $\mathrm{m}$ \\
\hline Qualität & 4 & & & & & \\
\hline Flexibilität & 3 & & & & & \\
\hline Humanität & 4 & & & & & \\
\hline$\ldots$ & $\ldots$ & & & & & \\
\hline Unternehmensziel $\mathrm{n}$ & 5 & & & & & \\
\hline \multicolumn{2}{|c|}{ Wichtigkeit der Kompetenzen } & 9 & 9 & 9 & $\ldots$ & 3 \\
\hline \multicolumn{2}{|c|}{ Eignung für ein mobiles Konzept } & & & & & \\
\hline
\end{tabular}

Bild 2. Gewichtung intendierter Kompetenzen durch Unternehmensziele (Anmerkung: Quantitative Angaben zur Bewertung dienen als Beispiel und entsprechen nicht den tatsächlichen Bewertungen) 


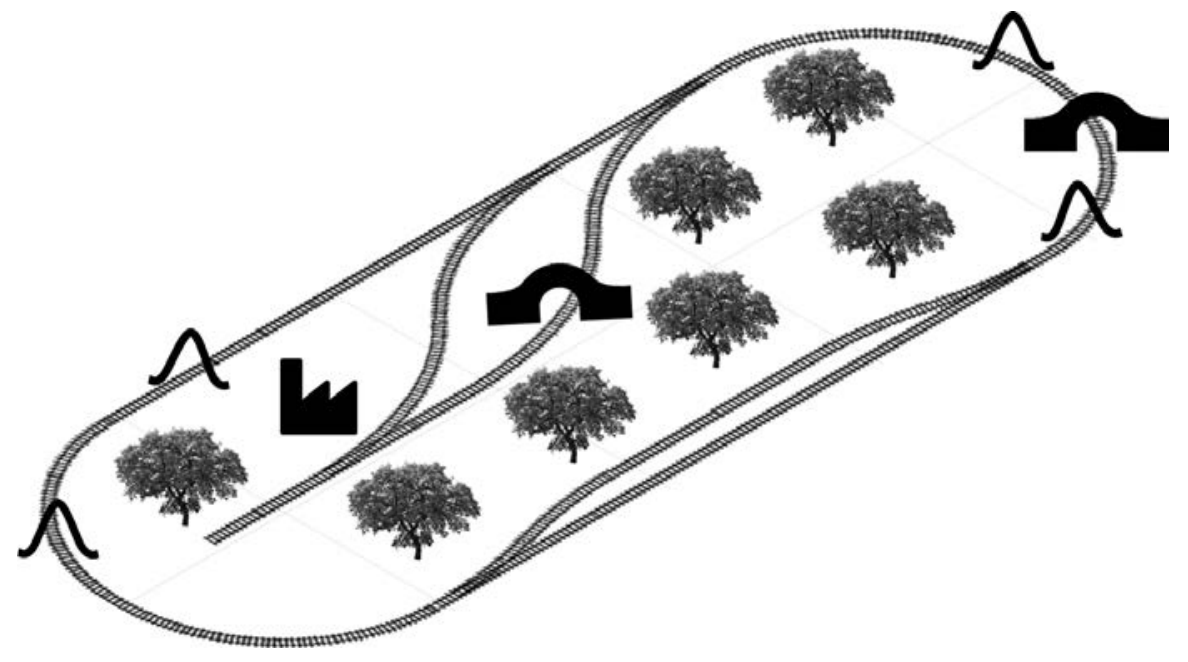

Bild 3. Gleisplan zum ersten Lernmodul

die Schnittstellen ihrer täglichen Arbeit $\mathrm{zu}$ weiteren beteiligten Personen und deren Aufgaben am Beispiel eines Ersatzschienenwechsels. Dazu werden Eingangsgrößen, beteiligte Personen sowie Aufgaben/Umfeld/Systeme in Form von Karten an die Teilnehmenden ausgeteilt. Die Karten sollen nun mithilfe einer Vorlage zu einem Systembild zusammengelegt werden. Die dazu stattfindenden Diskussionen dienen dem gewerkeübergreifenden Wissensaustausch und sind beabsichtigt. Der Unterrichtende gibt im Anschluss unterstützendes Feedback mithilfe einer Musterlösung. Die zur Entwicklung des Lernmoduls notwendigen Informationen stammen aus verschiedenen internen Arbeitsdokumenten der DB Netz AG sowie aus entsprechenden Fachabteilungen. Die Teilnehmenden erkennen anhand des Systembilds die Komplexität des gesamten Systems. Dem operativen Mitarbeitenden kann so die Vielzahl an beteiligten Personen sowie deren Aufgaben bewusst werden.

Darüber hinaus behandelt das dritte Lernmodul Themen aus dem Bereich Arbeitssicherheit. Die Teilnehmenden ordnen die zulässige persönliche Schutzausrüstung basierend auf ausgewählten Szenarien aus dem beruflichen Alltag zu. Dazu diskutieren sie in Teilgruppen, welche persönliche Schutzausrüstung zu den unterschiedlichen Szenarien zu tragen ist. Das Ergebnis wird im Anschluss präsentiert. Die Zuordnung der persönlichen Schutzausrüstung wurde mithilfe der Fachabteilung für Arbeitssicherheit erstellt. Die ordnungsgemäße persönliche Schutzausrüstung wird danach kurz vorgestellt. Des Weiteren ana- lysieren die Teilnehmenden mögliche Vermeidung anhand realer Unfälle im Unternehmen. Auch hier werden Teilgruppen gebildet, die nach der gruppeninternen Diskussion das Ergebnis vorstellen. Beide Teilübungen verdeutlichen die Relevanz der Arbeitssicherheit und vermitteln wichtige Erkenntnisse für den Alltag.

Zum Abschluss befragt der Unterrichtende die Teilnehmenden nach den wichtigsten Erkenntnissen des Tages und nach konkreten Maßnahmen für den individuellen Berufsalltag. Die Teilnehmenden haben die Möglichkeit, einen Partner auszuwählen, der sie nach circa zwei Wochen zur Umsetzung der Maßnahmen befragen und motivieren kann. Dies fördert den Transfer des Gelernten in den beruflichen Alltag.

Die drei Lernmodule können innerhalb eines Arbeitstags gemeinsam durchgeführt werden. Als Variante besteht die Möglichkeit diese unabhängig voneinander zu realisieren. Die potenziellen Teilnehmenden erhalten die Informationen zu den Lerninhalten und dem Anmeldeprozess durch ein umfangreiches Kommunikationskonzept.

\section{| Ausblick}

Die entwickelte mobile Lernfabrik wird in den sieben Regionalbereichen der DB Netz AG implementiert. Die Teilnahme ist freiwillig. Die zukünftigen Trainer werden dazu durch intensive Train-theTrainer-Veranstaltungen in jedem Regionalbereich geschult. Die mobile Lernfabrik stellt die erste von zwei Ausbaustufen dar. Im Forschungsprojekt zwischen Unfallursachen und Maßnahmen zur der DB Netz AG und der TU Darmstadt wird in der zweiten Stufe eine standortgebundene Lernfabrik für die DB Netz AG anhand eines zu entwickelnden Optimierungsmodells konfiguriert, aufbauend auf bisherigen Arbeiten [9]. Das zweistufige Verfahren bietet verschiedene Vorteile bei der Implementierung von Lernfabriken in Unternehmen: Zum einen werden Mitarbeitende so frühzeitig $\mathrm{zu}$ Weiterbildungsmaßnahmen informiert; zum anderen können Erkenntnisse und das Feedback aus bisherigen Durchläufen aus der ersten Stufe für die zweite genutzt werden. Die Ergebnisse validieren und erweitern zudem das am PTW entwickelte Vorgehen zur kompetenzorientierten Gestaltung von Lernfabriken [10].

\section{| Literatur}

1. Abele, E.; Metternich, J; Tisch, M.: Learning Factories - Concepts, Guidelines, Best-Practice Examples. Springer International Publishing, Basel 2018, S. 199-254 DOI: 10.1007/978-3-319-92261-4

2. Enke, J.; Kaiser, J.; Metternich, J.: Die Lernfabrik als Export-Erfolg. ZWF 112 (2017) 10, S. 644-647 DOI: $10.3139 / 104.111806$

3. Tisch, M.; Metternich, J.: Potentials and Limits of Learning Factories in Research, Innovation Transfer, Education, and Training. In: Proceedings of the $7^{\text {th }}$ CIRP-sponsored Conference on Learning Factories. Procedia Manufacturing (2017) 9, S. 89-96 DOI: 10.1016/j.promfg.2017.04.027

4. Abele, E.; Metternich, J.; Tisch, M.; Chryssolouris, G.; Sihn, W.; ElMaraghy, H.; Hummer, V.; Ranz, F.: Learning Factories for Research, Education, and Training. In: Proceedings of the $5^{\text {th }}$ Conference on Learning Factories. Procedia CIRP (2015) 32, S. $1-6$ DOI: 10.1016/j.procir.2015.02.187

5. Tisch, M.; Hertle, C.; Abele, E.; Metternich, J.; Tenberg, R.: Learning Factory Design: A Competency-oriented Approach Integrating Three Design Levels. International Journal of Computer Integrated Manufacturing 29 (2016) 12, S. 1355-1375 DOI: 10.1080/0951192X.2015.1033017

6. VDI - Verein Deutscher Ingenieure e. V.: VDI 2870-1: Ganzheitliche Produktionssysteme. Beuth Verlag, Berlin 2012

7. Anderson, L. W.: Krathwohl, D. R.; Airasian P.W.: A Taxonomy for Learning, Teaching and Assessing: A Revision of Bloom's Taxonomy of Educational Objectives. Longman, New York 2001, S.1-336

8. Enke, J.; Kraft, K.; Metternich, J.: Competency-oriented Design of Learning Modules. In: Proceedings of the $5^{\text {th }}$ Conference on Learning Factories. Procedia CIRP (2015) 32, S. 7-12 DOI: 10.1016/j.procir.2015.02.211 
9. Tisch, M.; Laudemann, H.; Kreß, A.; Metternich, J.: Utility-based Configuration of Learning Factories Using a Multidimensional, Multiple-choice Knapsack Problem. In: Proceedings of the $7^{\text {th }}$ CIRP-sponsored Conference on Learning Factories. Procedia Manufacturing (2017) 9, S. 25-32 DOI: 10.1016/j.promfg.2017.04.017

10. Tisch, M.: Modellbasierte Methodik zur kompetenzorientierten Gestaltung von Lernfabriken für die schlanke Produktion. Shaker Verlag, Aachen 2018, S. 1-295

\section{| Die Autoren dieses Beitrags}

Antonio Kreß, M.Sc., geb. 1989, studierte Wirtschaftsingenieurswesen mit der technischen Fachrichtung Maschinenbau an der Technischen Universität Darmstadt. Er ist seit 2017 Wissenschaftlicher Mitarbeiter am PTW in der Forschungsgruppe Center für industrielle Produktivität der Technischen Universität Darmstadt.
Nicole Lieb, Diplom-Pädagogin, studierte Erziehungswissenschaften mit dem Schwerpunkt Erwachsenenbildung und außerschulische Jugendbildung an der Universität Augsburg. Sie ist Senior-Referentin der Fachlichen Qualifizierung in der Zentrale der DB Netz AG und Projektleiterin für die Lernfabrik seitens der DB Netz AG.

Verena Lorenz, B.A., studierte Erziehungswissenschaften mit dem Schwerpunkt Erwachsenen- und Weiterbildung an der Universität Augsburg. Sie ist Trainee der Fachlichen Qualifizierung in der Zentrale der DB Netz AG und betreut die Entwicklung der mobilen Lernfabrik.

Prof. Dr.-Ing. Dipl.-Wirtsch.-Ing. Joachim Metternich, geb. 1968, ist Institutsleiter des Instituts für Produktionsmanagement, Technologie und Werkzeugmaschinen (PTW) an der Technischen Universität Darmstadt und leitet die Forschungsgruppen „Center für industrielle Produktivität“ und „Management industrieller Produktion“.

\section{Summary}

Competence-oriented Design of Mobile Learning Factory Modules at DB Netz AG. The idea for the conception of a mobile learning factory results from the motivation to develop an experiential and practical learning environment for the operative maintenance personnel. The article describes the competence-oriented design of mobile learning factory modules for DB Netz AG. For this purpose, three mobile learning factory modules were developed, which deal with the topics of work organisation, cross-discipline system observation and occupational safety. The underlying three-year research project is made possible by cooperation between DB Netz AG and the TU Darmstadt.

\section{Bibliography}

DOI 10.3139/104.112169

ZWF 114 (2019) 10; page 631 - 634

(C) Carl Hanser Verlag GmbH \& Co. KG ISSN 0032-678X 